\section{1) Watching single molecules in cells}

Examining cell biology at the singlemolecule level is methodologically challenging but offers high rewards.

In their quest to understand the function of biological systems some researchers have determined that classical ensemble measurements, by relying on averages, are missing spatial and temporal heterogeneity that could be crucial for understanding biological function. Now a new quest is on: to rectify this situation with experiments on single cells and single molecules.

Most single-molecule experiments, however, have been limited to in vitro experiments in which the object under study is removed from the cellular environment. This is often a necessity, but scientists are increasingly conducting singlemolecule experiments in whole cells.

One process being investigated using these methods is transcriptional activity. Over the past few years researchers developed several different methods capable of detecting single mRNA molecules in cells. These methods rely on a variety of different fluorescent probing techniques that push the limits of sensitivity, and they are beginning to produce valuable biological results that could not be obtained using classical ensemble measurements.

It turns out that transcription does not produce a steady stream of mRNA molecules as might be expected based on results from ensemble experiments. In fact, mRNA molecules are transcribed during large, random bursts of gene activity. Several groups discovered this in bacteria and yeast using GFP-based methods and Arjun Raj, Sanjay Tyagi and colleagues used highly optimized fluorescence in situ hybridization to extend and expand these results to mammalian cells (PLoS Biol. 4, e309; 2006). In 2007, Sunney Xie's laboratory extended single-molecule studies of gene expression in living cells to the measurement of transcription-factor binding to DNA (Science 316, 1191-1194; 2007).

\section{1) A meta-network of -omics}

Needed: a network of databases to comprehensively link current knowledge in the biosciences.

If the proverbial blind men were scientists, they would have described their respective parts of the elephant well. Its epigenome, genome, transcriptome, proteome and glycome would continually be analyzed with ever-increasing accuracy. A plethora of databases would make these findings easily accessible and encourage other researchers to contribute. And yet despite this detailed knowledge, we would still be a long way from understanding how all these parts act in concert to make an elephant.

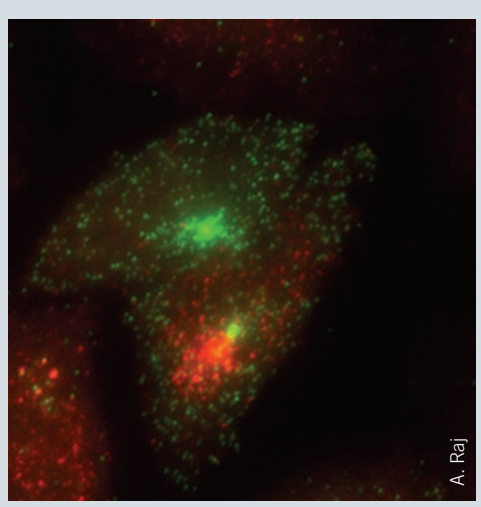

Single molecules of two different mRNAs imaged in cells.

The difficulties in conducting singlemolecule experiments in cells require the development of new methods though. The new super-resolution fluorescence microscopy techniques are certainly a step forward, but more developments are needed. We believe however, that the ability of single-molecule measurements to shine a light into the dark areas of biology that are invisible to traditional methods makes it a certainty that these methods will be developed in the future. Daniel Evanko

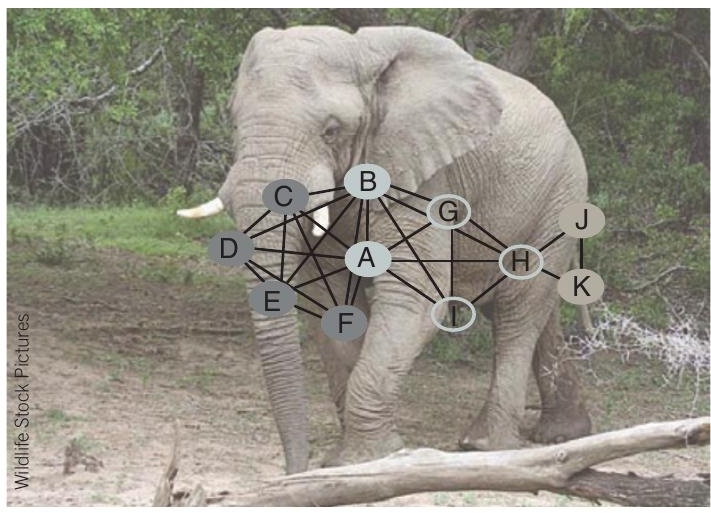

Integrating the parts to define the whole.

The challenge is to integrate knowledge gained in different areas to appreciate how each aspect contributes to the workings of a whole organism. The bottleneck to achieve this goal is no longer a lack of data, but the lack of ingenuity, computational means and financial support to integrate them in a 'meta-database'. Efforts toward this goal are certainly underway in many fields, evidenced, for example, by databases that connect clinical phenotypes with genome structure, gene sequence and expression profiles. To reach true depth of information, functional networks should integrate different approaches and, for example, enrich datasets on an interactome with genomic and proteomic data as well as cellular localization.

Let us allow ourselves to dream about the extent of information a true 'meta-database' could provide; a scientist starts a search with, for example, a protein of choice and with a few mouse clicks has a wealth of information at her disposal: gene sequence, epigenetic status, expression in different tissues, together with the whole proteome and interactome in that tissue, its functional role, the binding to other proteins, post-translational modifications, its role in disease, common mutations as well as available resources such as knock out animals, and more.

We have all the bits and pieces to realize this dream: what is needed is a way to link current databases into an easily accessible meta-network so people can optimally use knowledge generated outside their own box of expertise and integrate it all to decipher the big picture.

Nicole Rusk 